\title{
A lower bound on the local extragalactic magnetic field
}

\author{
Luis A. Anchordoqui and Haim Goldberg \\ Department of Physics, Northeastern University, Boston, MA 02115
}

\begin{abstract}
Assuming that the hard $\gamma$-ray emission of Cen $\mathrm{A}$ is a result of synchrotron radiation of ultrarelativistic electrons, we derive a lower bound on the local extragalactic magnetic field, $B>10^{-8}$ G. This result is consistent with (and close to) upper bounds on magnetic fields derived from consideration of cosmic microwave background distortions and Faraday rotation measurements.
\end{abstract}

Increasing our knowledge of the properties of extragalactic magnetic fields (EGM) on scales between Mpc and the visible horizon could significantly impact our understanding of phenomena in cosmic ray physics (propagation, dissipation via synchrotron radiation), cosmology (distortion of cosmic microwave background (CMB)), and particle physics (early universe primordial magnetogenesis during phase transitions). At present, surprisingly little is actually known about EGM: there are some measurements of diffuse radio emission from the bridge area between the Coma and Abell superclusters [1,2], which, under assumptions of equipartition allows an estimate of $\mathcal{O}(0.2-0.6) \mu \mathrm{G}$ for the magnetic field in this region. Such a large field may possibly be understood if the bridge region lies along a filament or sheet [3]. Faraday rotation measurements [4, 5. have thus far served to set upper bounds of $\mathcal{O}\left(10^{-9}-10^{-8}\right) \mathrm{G}$ on EGM on various scales [5.6], as have the limits on distortion of the CMB [7]8]. The Faraday rotation measurements sample EGM of any origin out to quasar distances, while the CMB analyses set limits on primordial magnetic fields [9]. We will discuss these bounds in some detail in the concluding section, when comparing with our results.

In this paper, we will use the results of a set of cosmic ray measurements taken over a ten-year period at the Sydney University Giant Air-shower Recorder (SUGAR) [10], and covering much of the southern sky, in order to set a lower bound on the size of the $B$-field between Earth and the nearby galaxy Centaurus A (Cen A), at a distance $d \approx 3.4 \mathrm{Mpc}$. The bound will be based on current models of dynamics in the "hot spots" of radiogalaxies, and these assumptions will be tested by future observations of ultra-high energy cosmic ray (CR) and high energy gamma ray fluxes from the direction of Cen A. We will initially outline the dynamical picture concerning the generation of high energy gamma rays. This picture leads to our Eq. (7), and then as a consequence to our lower bound on $B$. We may immediately note that we are aware of the crude angular and energy resolutions of the SUGAR observations. Our analysis will take into account these uncertainties.

Fanaroff-Riley II (FRII) galaxies [1] are the largest known dissipative objects (non-thermal sources) in the universe. Localized regions of intense synchrotron emission, so-called "hot spots", are observed within their lobes. These regions are presumably produced when the bulk kinetic energy of the jets ejected by a central active nucleus (supermassive black hole + accretion disk) is reconverted into relativistic particles and turbulent fields at a "working surface" in the head of the jets [12]. In what follows, we will adopt the first order Fermi shock acceleration mechanism in hot spots of FRII glaxies, as discussed in Refs. [13, 14], to account for particle acceleration to ultra high energies 15]. The applicability of this scenario to Cen A will be discussed in the next paragraph. The subtleties surrounding the conversion of particles' kinetic energy into radiation provide ample material for discussion [16 18]. The most popular mechanism to date relates $\gamma$-ray emission to the development of electromagnetic cascades triggered by secondary photomeson products that cool instanstaneously via synchrotron radiation 16, 17]. The characteristic single photon energy in synchrotron radiation emitted by an electron is

$$
E_{\gamma}=\left(\frac{3}{2}\right)^{1 / 2} \frac{h e E^{2} B}{2 \pi m_{e}^{3} c^{5}} \sim 5.4 B_{\mu \mathrm{G}} E_{19}^{2} \mathrm{TeV}
$$

For a proton this number is $\left(m_{p} / m_{e}\right)^{3} \sim 6 \times 10^{9}$ times smaller. Here, $B_{\mu \mathrm{G}}$ is the magnetic field in units of $\mu \mathrm{G}$ and $E_{19} \equiv E / 10^{19} \mathrm{eV}$. Thus, it is evident that high energy gamma ray production through proton synchrotron radiation requires very large $(\mathcal{O}(100 \mathrm{G}))$ magnetic fields.

We now discuss how the less luminous FRI galaxy Cen A fits into this picture. This radio-loud source $\left(l \approx 310^{\circ}\right.$, $b \approx 20^{\circ}$ ), identified at optical frequencies with the galaxy NGC 5128, is the closest example of the class of active galaxies [19]. Different multi-wavelength studies have revealed a rather complex morphology: it comprises a compact core, a jet also visible at $X$-ray frequencies, a weak counterjet, two inner lobes, a kpc-scale middle lobe, and two giant outer lobes. The jet would be responsible for the formation of the northern inner and middle lobes when interacting with the interstellar and intergalactic medium, respectively. There appears to be a compact structure in northern lobe, at the extrapolated end of the jet. This structure resembles the hot spots like those existing at the extremities of FRII galaxies. However, at Cen $\mathrm{A}$ it lies at the side of the lobe rather than at the most distant northern edge, and the brightness contrast (hot spot to lobe) is not as extreme [20].

In order to ascertain the capability of Cen A to accelerate particles to ultra high energies, one first applies 
the Hillas criterion 443] for localizing the Fermi engine in space, namely that the gyroradius $r_{g}=110 E_{20} / B_{\mu \mathrm{G}}$ kpc $\left(E_{20} \equiv E / 10^{20} \mathrm{eV}\right)$ be less than the size of the magnetic region. Low resolution polarization measurements in the region of the suspected hot spot give fields as high as $25 \mu \mathrm{G}$ 20. In certain of the regions where measurements at both high and low resolution are available, the $B$-field at high resolution can be twice that at low resolution. The higher resolution can reveal amplification in the post-shock region [22], yielding $B$-fields possibly as high as $50-60 \mu \mathrm{G}$ 20.23]. The radio-visible size of the hot spot can be directly measured from the large scale map of Ref. 24], giving $R_{\mathrm{HS}} \simeq 2 \mathrm{kpc}$. The actual size can be larger by a factor $\sim 2$ because of uncertainties in the angular projection of this region along the line of sight [25]. If one assumes that the magnetic field of the hot spot is confined to the visible region, then the limiting energy is $\sim 2 \times 10^{20} \mathrm{eV}$. However, it is plausible that the shock structure in the hot spot extends beyond the radio-visible region [14,26].

In light of this, we apply to Cen $\mathrm{A}$ the analyses of Refs. 14, 16], in which a limiting energy is obtained by balancing the characteristic time scale for diffusive shock acceleration

$$
\tau_{\mathrm{acc}} \simeq \frac{40}{\pi} \frac{1}{c \beta_{\mathrm{jet}}^{2}} \frac{1}{u}\left(\frac{E}{e B}\right)^{1 / 3} R^{-2 / 3}
$$

against the energy loss time scale

$$
\tau_{\text {loss }} \simeq \frac{6 \pi m_{p}^{4} c^{3}}{\sigma_{T} m_{e}^{2} B^{2}(1+A a)} E^{-1} .
$$

In the above, $\beta_{\text {jet }}=$ jet velocity in units of $c, u$ is the ratio of turbulent to ambient magnetic energy density in the region of the shock (of radius $R$ ), $B$ is the total magnetic field strength, $a$ is the ratio of photon to magnetic energy densities, $\sigma_{T}$ is the classical Thomson cross section, and $A$ is a measure of the relative strength of $\gamma p$ interactions against the synchrotron emission. In Ref. [14, $A$ is estimated to be $\approx 200$ almost independent of source. In Eq.(22) we assume that the turbulent component of the magnetic field follows a Kolmogorov spectrum with spectral index 5/3. Equating these characteristic times yields a value for the maximum proton energy

$$
E_{20}=1.4 \times 10^{5} B_{\mu \mathrm{G}}^{-5 / 4} \beta_{\mathrm{jet}}^{3 / 2} u^{3 / 4} R_{\mathrm{kpc}}^{-1 / 2}(1+A a)^{-3 / 4},
$$

where $R_{\mathrm{kpc}} \equiv R / 1 \mathrm{kpc}$. One can estimate $u \sim 0.4$ from the radio spectral index of synchrotron emission in the hot spot and the observed degree of linear polarization in the same region [27]. The jet velocity is model dependent, and estimates range from $\sim 500 \mathrm{~km} \mathrm{~s}^{-1}$ to $0.99 \mathrm{c}$ [20]. For FRI galaxies, $a$ is expected to be $\ll 1$ [16], and in our analysis we will sample a region of small $a$. In Fig. 1 we plot the relation between $\beta_{\text {jet }}$ and $a$ required to attain various energies, for fiducial values $B=60 \mu \mathrm{G}, R=4$ kpc. Since the range of values for $a$ and the jet velocity conform to expected values, it is plausible that Cen A can accelerate particles to energies $\gtrsim 10^{20} \mathrm{eV}$.

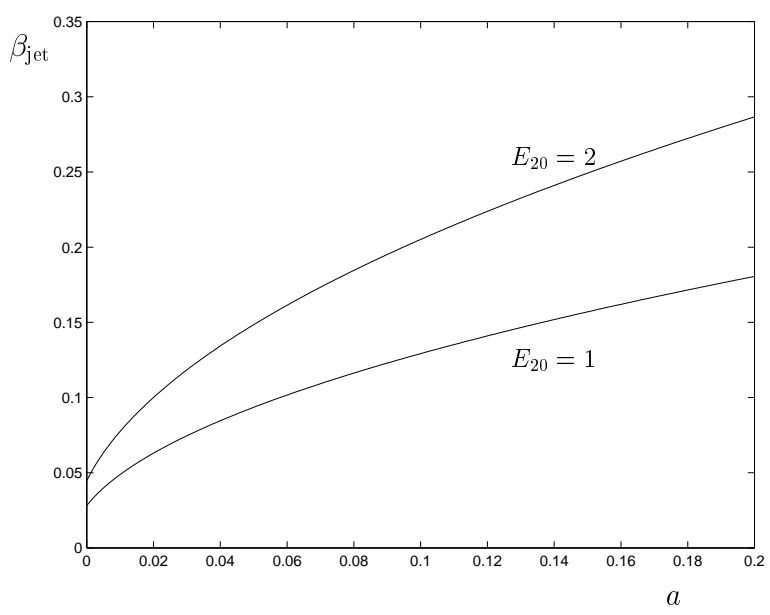

FIG. 1. Jet velocity as a function of parameter $a$ (defined in text), for different proton energies.

Recent observations of the gamma ray flux for energies $>100 \mathrm{MeV}$ (by the Energetic Gamma Ray Experiment Telescope (EGRET) [28]) allow an estimate $L_{\gamma} \sim 10^{41} \mathrm{erg} \mathrm{s}^{-1}$ for the source [29]. This value of $L_{\gamma}$ is consistent with an earlier observation of photons in the TeV-range during a period of elevated activity [30], and is considerably smaller than the estimated bolometric luminosity $L_{\mathrm{bol}} \sim 10^{43} \mathrm{erg} \mathrm{s}^{-1}[19]$. Data across the entire gamma ray bandwidth of Cen A is given in Ref. [31], reaching energies as high as $150 \mathrm{TeV}$ [32. Data at this energy await confirmation. For values of $B$ in the $\mu \mathrm{G}$ range, substantial proton synchrotron cooling is suppressed, allowing production of high energy electrons through photomeson processes. The average energy of synchrotron photons scales as $\bar{E}_{\gamma} \simeq 0.29 E_{\gamma}$ 33. With this in mind, it is straightforward to see that to account for $\mathrm{TeV}$ photons Cen A should harbor a population of ultra-relativistic electrons with $E \sim 6 \times 10^{18} \mathrm{eV}$. We further note that this would require the presence of protons with energies between one and two orders of magnitude larger, since the electrons are produced as secondaries [34].

There are plausible physical arguments [17,35 as well as some observational reasons [36] to believe that when proton acceleration is being limited by energy losses, the CR luminosity $L_{\mathrm{CR}} \approx L_{\gamma}$. In the spirit of [37, we introduce $\epsilon$, the efficiency of ultra high energy CR production compared to high energy $\gamma$ production - from the above, we expect $\epsilon \simeq 1$. Using equal power per decade over the interval $\left(E_{\min }, E_{\max }\right)$, we estimate a source luminosity

$$
\frac{E^{2} d N_{0}^{p+n}}{d E d t} \approx \frac{6.3 \epsilon L_{41} 10^{52} \mathrm{eV} / \mathrm{s}}{\ln \left(E_{\max } / E_{\min }\right)}
$$


where $L_{41} \equiv$ luminosity of Cen $\mathrm{A} / 10^{41} \mathrm{erg} \mathrm{s}^{-1}$ and the subscript "0" refers to quantities at the source.

For a relatively close source like Cen A, one can neglect interactions of cosmic ray protons with the universal background radiations. Consequently, the shape of the spectrum would be unmodified [38]. In order to discuss directionality, we first consider the case where there is no intergalactic magnetic field. Then en route to us the protons suffer no deflections and no spectral distortions. With the source luminosity (5), the counting rate at Earth in an energy bin $\left(E_{1}, E_{2}\right)$ for a detector of area $S$ due to Cen A would be

$$
\begin{aligned}
\left.\frac{d N}{d t}\right|_{B=0}= & \frac{S}{4 \pi d^{2}} \int_{E_{1}}^{E_{2}} \frac{E^{2} d N_{0}}{d E d t} \frac{d E}{E^{2}} \\
= & 14.3 \frac{\left(S / 100 \mathrm{~km}^{2}\right) \epsilon L_{41}}{\ln \left(E_{\max } / E_{\min }\right)} \\
& \times\left(\frac{1}{E_{1,20}}-\frac{1}{E_{2,20}}\right) \text { events/yr. }
\end{aligned}
$$

(Here $E_{1,20}=E_{1} / 10^{20} \mathrm{eV}$, etc.) This would all be concentrated in a cone of half-angle $\theta_{\text {res }}$, the observational resolution, about the direction of Cen $\mathrm{A}$.

As mentioned previously, the only existing measurements of ultrahigh energy cosmic rays arriving from directions which include Cen A were carried out at SUGAR during a ten year period 1968-1979. The array was located at $30^{\circ} 32^{\prime} \mathrm{S}, 149^{\circ} 46^{\prime} \mathrm{E}$, and presented a total area of $100 \mathrm{~km}^{2}$. Shown as asterisks in Fig. 2, as given in Ref. [10], are the arrival directions of 80 events with energies above $4 \times 10^{19} \mathrm{eV}$. The direction of Cen A is indicated with a five-pointed star, and the experimental $1 \sigma$ uncertainties in arrival directions of events near the Cen A direction are indicated by dashed lines [39]. The solid line ovals surrounding Cen $\mathrm{A}$ indicate regions within $10^{\circ}$ and $25^{\circ}$, respectively, of Cen $\mathrm{A}$, and their significance will be explained shortly.

We now obtain the $B=0$ event rate expected at SUGAR in the direction of Cen A, as predicted by Eq.(6). The energy bin is appropriate to the data of [10], $E_{1,20}=0.4, E_{2,20}=2$. A conservative lower bound on the energy of protons which are progenitors of high energy gamma radiation is $E_{\min }=1 \times 10^{19} \mathrm{eV}$. It also reasonable to take for the upper bound $E_{\max }=4 \times 10^{20}$ $\mathrm{eV}$ [40]. With this input, we obtain from Eq.(6)

$$
\left.\frac{d N}{d t}\right|_{B=0} \gtrsim 8 \epsilon L_{41} \text { events/year. }
$$

In the preceding discussion we have indicated that under plausible conditions may expect $\epsilon \simeq 1$. However, even if $\epsilon L_{41}$ is as small as 0.1, we may, in the absence of a magnetic field, expect 8 events in 10 years from the direction of Cen A.

The cosmic ray orbits undergo bending in both the Galactic and extragalactic magnetic fields. The magnetic deflection of protons in the Galactic disk has been studied in detail in 411. This analysis includes two extreme options for the behavior of the field, reflecting the different symmetries with respect to field reversals in the $r$ and $z$-directions. The $B$-field has a $1 / r$ behavior, with deviations calculated out to $20 \mathrm{kpc}$ from the Galactic center. The r.m.s. deviation (averaged over arrival direction), for an energy of $4 \times 10^{19} \mathrm{eV}$, varies from $7.9^{\circ}$ to $10.5^{\circ}$ in going between the two models. This deviation shows an approximately linear decrease with increasing energy. All the events which we will consider have energies $>4 \times 10^{19} \mathrm{eV}$, so that conservatively, we allow an uncertainty of $10^{\circ}$ in arrival direction due to deviation in the Galactic magnetic field. Events within the inner oval of Fig. 2 represent events which could have originated from Cen A, and have suffered deflection only in the Galactic field. It is clear that at most 1 event can be categorized in this manner, so that in order to have at least 8 events (see previous paragraph), some amount of extragalactic field is necessary.

We treat the extragalactic deviation in a standard manner 42]. With $B_{\mathrm{nG}}=B / 10^{-9} \mathrm{G}$, the Larmor radius of a particle in this field is $r_{\mathrm{L}} \simeq 10^{2} \mathrm{Mpc} E_{20} / B_{\mathrm{nG}}$. If this is sizeably larger than the coherence length of the magnetic field $\ell_{\text {coh }}$, the typical deflection angle from the direction of the source, located at a distance $d$, can be estimated assuming that the particle makes a random walk in the magnetic field 42

$$
\theta(E) \simeq 0.54^{\circ}\left(\frac{d}{1 \mathrm{Mpc}}\right)^{1 / 2}\left(\frac{\ell_{\mathrm{coh}}}{1 \mathrm{Mpc}}\right)^{1 / 2} \frac{B_{\mathrm{nG}}}{E_{20}} .
$$

Thus, in the case that $\theta(E) \ll 1$, we expect that the counting rate (6) would be spread out over a cone of half-angle

$$
\bar{\theta}(E) \simeq\left(\theta(E)^{2}+\theta_{\mathrm{res}}^{2}\right)^{1 / 2} .
$$

We now draw a second oval in Fig. 2 to include the least number of events compatible with the expectation from Cen A. This second oval contains (or partially contains) 7 events within $25^{\circ}$ of Cen A. From our previous discussion of the Galactic effects, this leads us to conclude that cosmic rays with $E>4 \times 10^{19} \mathrm{eV}$ experience a deviation of at least $15^{\circ}$ in extragalactic magnetic fields during their transit from Cen A to Earth.

In conjunction with Eqs. (8) and (9) we utilize this conclusion to obtain a lower bound on the $B$-field between Earth and Cen A. The average energy $\bar{E}$ of the cosmic ray events above $4 \times 10^{19} \mathrm{eV}$, as reported in [10], is $6.3 \times 10^{19}$ $\mathrm{eV}$. Requiring $\bar{\theta}(\bar{E})>15^{\circ}$, with $\theta_{\text {res }}=3^{\circ}$ and $d=3.4$ $\mathrm{Mpc}$, we obtain from (8) and (9)

$$
B>9.5 \times 10^{-9}\left(\ell_{\mathrm{coh}} / 1 \mathrm{Mpc}\right)^{-1 / 2} \mathrm{G} .
$$

Since the coherent length is most likely $<1 \mathrm{Mpc}$, we obtain the bound stated in the abstract,

$$
B>1.0 \times 10^{-8} \mathrm{G} \text {. }
$$




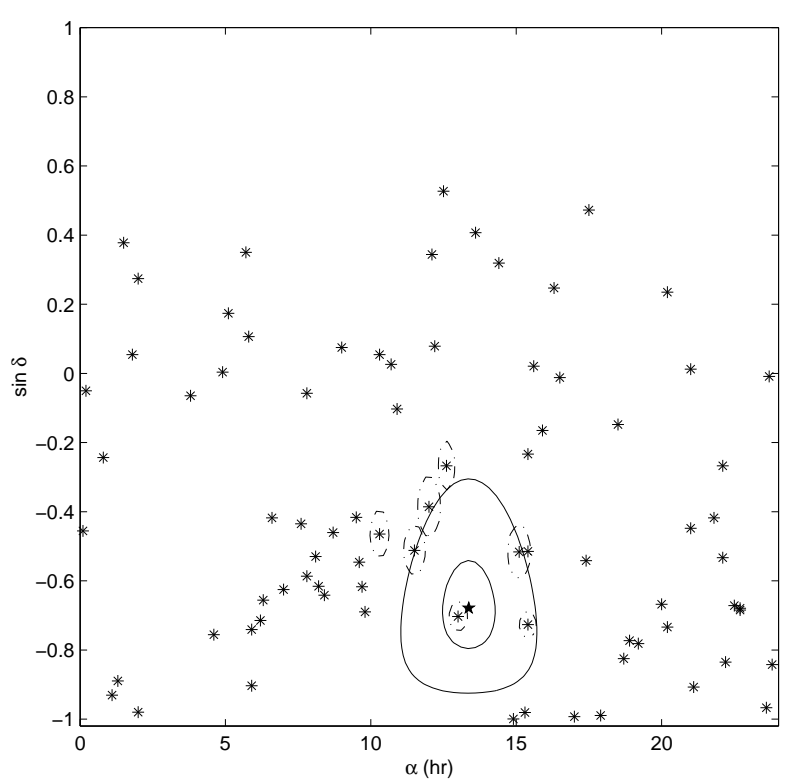

FIG. 2. The nominal arrival directions ( $\alpha=$ right ascension, $\delta=$ declination) of SUGAR events with energies above $4 \times 10^{19} \mathrm{eV}$. Also shown in solid lines are contour maps indicating the circular areas of the celestial sphere centered at Cen A (indicated by $\star$ ) with $10^{\circ}$ and $25^{\circ}$ radii. The dashed lines surrounding several of the events indicate the angular resolution of the experiment.

Our analysis has utilized the SUGAR data with energies assigned according to the Hillas model 43. With this prescription, the energy spectrum agrees with that of other experiments [10]. An alternate method [44] which underestimates the energy by a factor of more than 2 leads to a spectrum which does not show this agreement [10]. The lower energy would have two effects in our analysis: $(i)$ the expected event rate (7) would double because of the smaller lower limit on the energy bin $(i i)$ the deviation in the Galactic magnetic field would roughly double 41], leading to an increase in the size of the inner oval in Fig. 2. Following the same procedure as previously, and still utilizing the conservative bound $\epsilon L_{41} \geq 0.1$, we would find that $B>5 \mathrm{nG}$. However, because there is general consensus 45] that the spectral evidence strongly favors the Hillas energy normalization, we maintain our bound as $B>10 \mathrm{nG}$ 46.

The limit (11) has an immediate implication with respect to lower energy cosmic rays: from Eq.(8), one can conclude that if $B>10 \mathrm{nG}$ all directionality is lost for protons with energies below $\sim 2 \times 10^{19} \mathrm{eV}$. This is consistent with the absence of any anisotropy in the events observed at SUGAR at these lower energies [47.

We turn to discuss our result in light of considerations which impose upper bounds on magnetic fields. (1) An analysis of $\mathrm{He}^{4}$ production provides a weak bound $B_{0}<10^{-6} \mathrm{G}$ on a homogeneous primordial field [48; however, such fields are largely dissipated prior to nucleosynthesis 49]. (2) From limits on distortions of the cosmic microwave background (CMB), there is an up- per bound $B_{0}<3.4 \times 10^{-9} \mathrm{G}$ for fields homogeneous on present horizon scales [7]. Our lower bound (11) exceeds this, so that we may conclude that the magnetic field from here to Cen A contains components in excess of a homogeneous relic background field. (3) The analysis of CMB distortions extended to inhomogeneous fields provides a larger bound $B_{0}<3 \times 10^{-8} \mathrm{G}$ on scales between $400 \mathrm{pc}$ and $0.6 \mathrm{Mpc}$ ]. This lies above our limit, so that the local EGM is consistent with being of primordial origin. (4) The absence of a positive signal in Faraday rotation measurements on QSO's [5] provides upper limits on magnetic fields (of any origin) as a function of reversal scale. These bounds depend significantly on assumptions about the electron density profile as a function of red shift $z$. When electron densities follow that of the Lyman- $\alpha$ forest, the average magnitude of the magnetic field receives an upper limit of $B<10^{-9} \mathrm{G}$ for reversals on the scale of the horizon, and $B<10^{-8} \mathrm{G}$ for reversal scales on the order of $1 \mathrm{Mpc}$ [6]. The latter upper bound is roughly coincident with our lower limit in our galactic neighborhood. Local fluctuations in electron densities on scales of 1-30 Mpc can lead to very large concommitant fluctuations in the corresponding magnetic field [3], so that the bound in [6] should be read as averaged over many reversal cells between Earth and the light sources at distances out to $z=2.5$. If it should happen that the fluctuations in the magnitude of $B$ in the Mpc cells out to the horizon are of the same order as $B_{\text {avg }}$ itself, then our result can imply that the average value of the intergalactic magnetic field is of the order of $10^{-8} \mathrm{G}$. Because of the $B^{2}$ dependence of synchrotron radiation loss by secondary electrons, a field of this strength can have important implication on the development of electromagnetic cascades. Such cascades are characteristically associated with the decay of supermassive relic particles or topological defects. An average magnetic field of $10^{-8} \mathrm{G}$ will impose strong constraints on "top-down" models [50].

The SUGAR observations used in this analysis were recorded more than twenty years ago. Both resolution and statistics will be vastly improved with data to be available from facilities which will observe the southern sky. These are now coming on line (Auger [51]), or are expected to (EUSO/OWL [52]) in the not-too-distant future. These data will significantly enhance our knowledge of magnetic field strengths in the extragalactic neighborhood of the Milky Way.

We would like to thank Peter Biermann for sharing with us his expertise about magnetic fields in shock environment. This work was partially supported by CONICET (LAA), and the National Science Foundation (HG). 
[1] K.-T. Kim, P. P. Kronberg, G. Giovannini and T. Venturi, Nature 341, 720 (1989).

[2] Fields of $\mathcal{O}(\mu \mathrm{G})$ are also indicated in a more extensive study of 16 low redshift clusters. T. E. Clarke, P. P. Kronberg and H. Böhringer, Astrophys. J. 547, L111 (2001).

[3] D. Ryu, H. Kang, and P. L. Biermann, Astron. Astrophys. 335, 19 (1998).

[4] J. P. Vallée, Astrophys. J. 360, 1 (1990).

[5] P. K. Kronberg, Rep. Prog. Phys. 57, 325 (1994).

[6] P. Blasi, S. Burles, and A. V. Olinto, Astrophys. J. Lett. 512, L79 (1999).

[7] J. D. Barrow, P. G. Ferreira, and J. Silk, Phys. Rev. Lett. 78, 3610 (1997).

[8] K. Jedamzik, V. Katalinić, and A. V. Olinto, Phys. Rev. Lett. 85, 700 (2000).

[9] For a recent survey, see R. Maartens, Pramana 55, 575 (2000), astro-ph/0007352. See also P. L. Biermann, Cosmic Magnetic Fields, Erice lecture, December 2000.

[10] M. M. Winn et al., J. Phys. G 12, 653 (1986).

[11] B. L. Fanaroff and J. M. Riley, Mon. Not. R. Astron. Soc. 167, 31P (1974).

[12] R. D. Blandford and M. J. Rees, Mon. Not. R. Astron. Soc. 169, 395 (1974).

[13] L. O'C. Drury, Rept. Prog. Phys. 46, 973 (1983).

[14] J. P. Rachen and P. L. Biermann, Astron. Astrophys. 272, 161 (1993).

[15] The efficiency of the first order Fermi mechanism for accelerating particles has been recently questioned (see, e.g., M. Ostrowski and J. Bednarz, astro-ph/0101069, and references therein).

[16] P. L. Biermann and P. A. Strittmatter, Astrophys. J. 322, 643 (1987).

[17] K. Mannheim, Astron. Astrophys. 269, 67 (1993); Space Sci. Rev. 75, 331 (1996); Science 279, 684 (1998); Rev. Mod. Astron. 12, 101 (1999).

[18] F. A. Aharonian et al., Astrophys. J. 543, L39 (2000).

[19] F. P. Israel, Astron. Astrophys. Rev. 8, 237 (1998).

[20] J. O. Burns, E. D. Feigelson and E. J. Schreier, Astrophys. J., 273, 128 (1983).

[21] A. M. Hillas, Ann. Rev. Astron. Astrophys. 22, 425 (1984).

[22] L. Landau and E. Lifchitz, Fluid Mechanics (Pergamon Press, Oxford, 1958).

[23] G. E. Romero, J. A. Combi, S. E. Perez Bergliaffa and L. A. Anchordoqui, Astropart. Phys. 5, 279 (1996).

[24] N. Junkes, R. F. Haynes, J. I. Harnett, and D. L. Jauncey, Astron. Astrophys. 269, 29 (1993).

[25] For example, an explanation of the apparent absence of a counterjet in Cen A via relativistic beaming suggests that the angle of the visible jet axis with respect to the line of sight is at most $36^{\circ}$ 20, which could lead to a doubling of the hot spot radius. It should be remarked that for a distance of $3.4 \mathrm{Mpc}$, the extent of the entire source has a reasonable size even with this small angle.

[26] Simulations of the magnetic field in shock environments reveal a complex Mach-disk and extended oblique shock structure. Therefore, it is necessary to consider the entire shock environment for a complete picture of particle acceleration, confinement, and transport. Semi-realistic simulations of such structures, which include energetic particles and magnetic fields, are just beginning to be available. (P. Biermann, private communication).

[27] J. A. Combi and G. E. Romero, Astron. Astrophys. Suppl. 121, 11 (1997).

[28] P. Sreekumar et al., Astropart. Phys. 11, 221 (1999).

[29] Note that the received radiation is negligibly affected by interactions with the various radiation backgrounds, F. A. Aharonian, New Astron. 5, 377 (2000).

[30] J. E. Grindlay et al., Astrophys. J. 197, L9 (1975).

[31] H. Steinle, astro-ph/0105482.

[32] R. W. Clay, B. R. Dawson and R. Meyhandan, Astropart. Phys. 2, 347 (1994).

[33] V. L. Ginzburg, S. I. Syrovatsky, Ann. Rev. Astron. Astrophys. 3, 297 (1965).

[34] Consecutive factor of 2 energy losses occur in the processes $p \gamma \rightarrow N \pi^{0}, \pi^{0} \rightarrow \gamma \gamma, \gamma \rightarrow e^{+} e^{-}$. Eq.(1) then implies proton energies of $\sim 10^{20} \mathrm{eV}$ for $100 \mathrm{TeV}$ photons.

[35] See E. Waxman, Phys. Rev. Lett. 75, 387 (1995), for a nice discussion of this point in the context of GRBs.

[36] S. Rawlings and R. Saunders, Nature 349, 138 (1991).

[37] G. R. Farrar and T. Piran, astro-ph/0010370; L. A. Anchordoqui, H. Goldberg, and T. J. Weiler, Phys. Rev. Lett. 87, 081101 (2001) astro-ph/0103043.

[38] See for instance, F. A. Aharonian, and J. W. Cronin, Phys. Rev. D 50, 1892 (2000).

[39] This is given in Ref. [10] as $\theta_{\text {res }}=3^{\circ} \sec \theta_{\text {zenith. The sev- }}$ eral events at declinations $\delta$ near $-90^{\circ}$ have small errors in the $\delta$ direction, and do not intersect the oval regions.

[40] Our results are insensitive to lowering $E_{\max }$ to $2 \times 10^{20}$ $\mathrm{eV}$, or lower.

[41] T. Stanev, Astrophys. J. 479, 290 (1997).

[42] E. Waxman and J. Miralda-Escudè, Astrophys. J. Lett. 472, L89 (1996).

[43] M. A. Hillas et al., Proc. 12th Int. Conf. on Cosmic Rays, Hobart 3, 1007 (1971).

[44] L. Goorevich and L. S. Peak, J. Phys. G. 1, 762 (1975).

[45] L. J. Kewley, R. W. Clay and B. R. Dawson, Astropart. Phys. 5, 69 (1996).

[46] A lower cutoff on the maximum attainable energy would increase the expected event rate, and would decrease the possible observed events, leaving the bound unchanged.

[47] M. M. Winn et al., J. Phys. G 12, 675 (1986).

[48] B. Cheng, A. V. Olinto, D. Schramm, and J. Truran, Phys. Rev. D 54, 4714 (1996); P. Kernan, G. Starkman, and V. Vachaspati, Phys. Rev. D 54, 7202 (1996); D. Grasso and H. R. Rubinstein, Phys. Lett. B 379, 73 (1996).

[49] K. Jedamzik, V. Katalinić, and A. V. Olinto, Phys. Rev. D 57, 3264 (1998).

[50] For a review of "top-down" models, see P. Bhattacharjee and G. Sigl, Phys. Rep. 327109 (2000). The effects of magnetic fields on the electromagnetic cascade are discussed in R. J. Protheroe and P. A. Johnson, Astropart. Phys. 4, 253 (1996); R. J. Protheroe and T. Stanev, Phys. Rev. Lett. 77, 3708 (1996), [Erratum-ibid 78, 3420 (1997)].

[51] D. Zavrtanik, Nucl. Phys. B Proc. Suppl. 85, 324 (2000).

[52] http://ifcai.pa.cnr.it/euso.html, http://owl.gsfc.nasa.gov 